not statistically significant. No association was significant in Bayesian analyses.

Conclusions We suggest that future studies use similar biologically-based exposure assessments in order for us to be sure what advice we should give to the millions of women around the world who work at night.

\section{MELATONIN AND SEX HORMONE BIOMARKERS AND LIGHT INTENSITY EXPOSURE IN FEMALE AND MALE PERMANENT NIGHT SHIFT WORKERS}

${ }^{1} \mathrm{~K}$ P Papantoniou, ${ }^{2}$ Pozo, ${ }^{1}$ Espinosa, ${ }^{3}$ Middleton, ${ }^{3}$ Skene, ${ }^{1}$ Castaño-Vinyals. ${ }^{1}$ Centre for Research in Environmental, Epidemiology (CREAL), Barcelona, Spain; ${ }^{2}$ IMIM (Hospital del Mar Medical Research Institute), Barcelona, Spain; ${ }^{3}$ Chronobiology, University of Surrey, Guildford, United Kingdom

\subsection{6/oemed-2013-101717.206}

Objectives Night shift work has been associated with higher breast cancer risk. It has been proposed that night shift workers experience light-induced reduction in melatonin production. Melatonin has direct oncostatic properties and a potential interplay with reproductive hormones. In this cross-sectional study the hypothesis was that night shift workers produce less melatonin and more estrogens and androgens compared to day workers. Changes in the rhythm of hormone production under different individual light exposures were evaluated.

Methods 75 permanent night workers and 42 day workers of both sexes, aged 22-64 years, were recruited from 4 companies in Barcelona, Spain. Levels of 6-sulfatoxymelatonin (melatonin metabolite) and 27 steroid metabolites were measured in urine samples collected from all voids over 24-hours on a working day by all participants. Simultaneously participants wore a data logger that continuously recorded their light exposure. Sociodemographic, occupation and lifestyle information was collected for each participant by interview. Cosinor analysis was performed for 6-sulfatoxymelatonin in every individual to evaluate their circadian rhythm estimating the mesor (midpoint in the full-range), amplitude (difference of the peak value to the mesor) and peak time of production. Geometric means were calculated for each parameter in night and day workers.

Results Sociodemographic and lifestyle characteristics of day and night shift groups were not significantly different. 6-sulfatoxymelatonin production was significantly lower in night compared to day workers ( mesor 10.9 vs $14.2 \mathrm{ng} / \mathrm{mg}$ creatinine respectively; amplitude 11.5 vs $18.3 \mathrm{ng} / \mathrm{ml}$ creatinine) and peak time was later in night shift workers (6:00 am vs 3:48 am). Mean oestrogen and androgen levels tended to be higher among night workers but differences were not significant.

Conclusions This study indicates potential differences in melatonin and steroid profiles between night and day workers. Results from hormone levels in relation to personal light exposure using nonlinear mixed models will be presented.

\section{NIGHTSHIFT WORK AND LEVELS OF 6-SULFATOXYMELATONIN, CORTISOL AND SEX HORMONES IN MEN}

${ }^{1} \mathrm{P}$ B Parveen, ${ }^{2}$ Mirick, ${ }^{2}$ Davis. ${ }^{1}$ Seattle, United States of America; ${ }^{2}$ Fred Hutchinson Cancer Research Center, Seattle, WA, United States of America

10.1136/oemed-2013-101717.207

Objectives Nightshift work has been associated with cancer among men, but the mechanism underlying this association is not clear. We investigated whether male nightshift workers demonstrated changes in the normal circadian levels and secretion patterns of melatonin, cortisol and sex hormones that may be directly related to cancer risk.

Methods Participants were 185 male nightshift workers (NSW) and 158 male dayshift workers (DSW) employed as healthcare providers, aged 22-55. Urine samples were collected throughout work and sleep periods and assayed for various hormone metabolites.

Results Compared to DSW during their nighttime sleep, NSW had significantly lower levels of 6-sulfatoxymelatonin during daytime sleep, nighttime work, and nighttime sleep on their offnights $(57 \%, 62 \%$ and $40 \%$ lower, respectively). Urinary cortisol levels in the NSW were $16 \%$ higher during daytime sleep and $13 \%$ lower during nighttime sleep on off-nights, compared to DSW during nighttime sleep. While cortisol levels between NSW during night work and DSW during night sleep were not significantly different, metabolites of cortisol (e.g. cortisone, tetrahydrocortisol) were significantly increased among NSW. No significant differences were observed in testosterone or dihydrotestosterone levels between nightshift workers during their day sleep or night sleep compared to dayshift workers during nighttime sleep.

Conclusions Male sex hormones have been implicated in prostate carcinogenesis, however, results of this study indicate that the impact of nightshift work on cancer risk may occur through other mechanisms. Substantially reduced 6-sulfatoxymelatonin levels during night work, daytime sleep and even night sleep on off-nights among night shift workers were observed, and given the oncostatic properties of melatonin, this chronic reduction in melatonin among nightshift workers may represent an important carcinogenic mechanism. Corticosteroid secretion and metabolism was also found to be impacted by night shift work, which could have implications for cancer risk through its effects on immune function.

\section{Session: Plenary session}

\section{CHANGES IN EMPLOYMENT CONDITIONS AND MENTAL HEALTH DURING THE ECONOMIC CRISIS IN MIGRANT WORKERS IN SPAIN}

${ }^{1} G$ R R Robert Roca, ${ }^{2}$ Ronda Pérez, ${ }^{1}$ Martínez Martínez, ${ }^{1}$ Felt, ${ }^{1}$ García Benavides. ${ }^{1}$ Universitat Pompeu Fabra, Barcelona, Spain; ${ }^{2}$ Universidad de Alicante, Alicante, Spain

\subsection{6/oemed-2013-101717.208}

Objective Evaluate the influence of changes in employment conditions on mental health of migrant workers in Spain, in the context of economic crisis.

Methods Follow-up survey at two time points: 2008 and 2011, whose reference population consists of 318 workers from Colombia, Ecuador, Morocco and Romania living in Spain. Those who reported good mental health $(n=214)$ at 2008 were selected to evaluate, after three years, the incidence of poor mental health according to several sociodemographic and occupational factors (sex, age, nationality, education level, occupation and employment status), and the association between this health indicator and different employment trajectories during this period, by means of adjusted odds ratio (aOR).

Results There is an increased risk of poor mental health in those workers who lost their employment $(\mathrm{aOR}=3.62$; IC 95\%: 1.64-7.96), who increased the number of work hours 
$(\mathrm{aOR}=2.35 ;$ IC 95\%: 1.02-5.44), whose monthly income decreased $(\mathrm{aOR}=13.61$; IC 95\%: 1.49-124.35) or persisted in the lowest income range $(\mathrm{aOR}=14.56$; IC 95\%: 1.46-144.89). This was also the case for those workers who continued their temporary documented $(\mathrm{aOR}=5.05$; IC 95\%: 1.39-18.34) or undocumented $(\mathrm{aOR}=36.59$; IC 95\%: 2.47-541.17) status. A decreased risk was observed in workers who became affiliated to the Social Security (aORa $=0.10$; IC 95\%: 0.02-0.48).

Conclusions There is an increase of poor mental health in immigrant workers in Spain related to the worsening of their employment conditions. At the same time, it was observed a decrease when Social Security protection increased.

\section{AFTER HURRICANE SANDY, IMPLEMENTATION OF THE EXPANDED SYNDROMIC SURVEILLANCE FOR RECOVERY WORKERS AND RESIDENTS}

${ }^{1}$ H K Kim, 'Liu, ${ }^{2}$ Tak, 'Dropkin, 'Moline, ' ${ }^{1}$ Silverman. 'Hofstra North Shore-LIJ School of Medicine, Great Neck, NY, United States of America; ${ }^{2}$ Massachusetts Department of Public Health, Boston, United States of America

\subsection{6/oemed-2013-101717.209}

Objectives Hurricane Sandy brought wide spread devastation in the region of New York and New Jersey in USA. To our knowledge, there have been no official reports of the health effects from Hurricane Sandy since the day of Hurricane arrival 10/29 in 2012.

Methods To rapidly assess the health impacts of Hurricane Sandy among recovery workers and residents in the affected area, we are building an expanded syndromic surveillance system that integrates the near real-time electronic Emergency Department (ED) visit records collected from 14 EDs within a large health system in NY, the air quality index data from the Environmental Protection Agency (EPA), and the weather data from the National Oceanic and Atmospheric Administration (NOAA). Recovery workers will be identified from billing database. Approximately $50 \%$ of occupational/industrial information of ED patients are missing, however patients with work-related injuries and illnesses required to provide this information according to Worker's Compensation system in US. Through a multilevel case-crossover design, we aim to rapid identification of elevated health effects during four exposure windows we developed (the Pre-, During-, Short-term, and Long-term PostHurricane) by comparing ED records before (from 2005) and after Hurricane Sandy while adjusting for air pollution levels and weather conditions. The continuing monitoring during the fourth exposure window (i.e. Long-term Post- Hurricane) will ensure early detection of potential occurrences of chronic diseases stemmed from the initial disaster-related acute forms. The implementation of the syndromic surveillance within a large health system will not only improve the healthcare delivery, but also provide important information to the outside stakeholders such as public health agencies to enhance strategic planning for rapid post-disaster response.

\section{ATTENUATION OF EXPOSURE EFFECTS OVER TIME: A SIMULATION STUDY}

${ }^{1} \mathrm{~K}$ Steenland, ${ }^{2}$ Karnes, ${ }^{2}$ Barry, ${ }^{2}$ Darrow. ${ }^{1}$ Atlanta, United States of America; ${ }^{2}$ Emory U, Atlanta, Ga, United States of America

10.1136/oemed-2013-101717.210
Background Positive exposure-response trends often diminish at higher cumulative exposure, correlated with longer follow-up time. Depletion of susceptibles, increased measurement error at higher cumulative exposure, and saturation of biological pathways, have all been postulated as reasons for attenuation.

Methods We conducted simulations to evaluate rate ratios over time under different assumptions about susceptibility to exposure effects and measurement error; we evaluated exposure-response trends to determine whether attenuation was evident. We simulated a dynamic cohort in which entry occurred over time; the metric of interest was duration of exposure. We also considered cross-sectional analyses in which follow-up started only after a certain point of time. Simulations considered 10,000 subjects enrolled from 1940-2010 and followed through 2020. Ten simulations were conducted for each scenario and exposureresponse parameters averaged. An excess relative risk model was used to generate the relationship between duration of exposure and disease, controlling for age. Measurement error of both classical and Berkson type were simulated, with increasing error with increasing exposure. Cox regression was used to evaluate exposure-response trends.

Results Under all scenarios considered with less than 100\% susceptibility among the exposed, there was evidence of depletion of susceptibles over follow-up time. However, under realistic scenarios considered here, there was only modest evidence of attenuation of a linear exposure-response trend due to depletion of susceptibles. Classical measurement error, but not Berkson error, produced attenuation. Cross-sectional analyses did not dramatically change attenuation patterns.

Conclusions Marked attenuation of exposure-response trends over follow-up time is more likely due saturation of biological pathways or, perhaps less likely, to classical measurement error than to either the depletion of susceptibles or Berkson measurement error.

\section{A NEW OCCUPATIONAL CANCER: CHOLANGIOCARCINOMA AMONG OFFSET COLOR PROOF-PRINTING WORKERS EXPOSED TO 1,2- DICHLOROPROPANE AND/OR DICHLOROMETHANE}

${ }^{1} \mathrm{~S}$ K Kumagai, ${ }^{2}$ Kurumatani. 'University of Occupational and Environmental Health, Kitakyushu, Japan; ${ }^{2}$ Nara Medical University School of Medicine, Kashihara, Japan

\subsection{6/oemed-2013-101717.211}

Objectives At the 85th Annual Meeting of the Japan Society for Occupational Health (31 May, 2012, Nagoya, Japan), we reported a cluster of five patients with cholangiocarcinoma among workers in the offset colour proof-printing section of a small printing company in Osaka, Japan. This was the first report of occupational cholangiocarcinoma in the world. Further investigation led to the identification of a total of 11 patients (Occup Environ Med 2013, in print). Here, we present an epidemiological characteristic of the workers in the printing section.

Methods We identified 62 men who had worked in the proofprinting section for at least one year between 1991 and 2006. We interviewed them to learn about the chemicals they used, and estimated their chemical exposure levels. We also investigated medical records of the 11 patients and calculated the standardised mortality ratio (SMR) from 1991 to 2011.

Results Workers used 1,2-dichloropropane (1,2-DCP) from approximately 1985 to 2006 and dichloromethane (DCM) from approximately 1985 to $1997 / 98$. Exposure concentrations were estimated to be 100-670 ppm for 1,2-DCP and 80-540 ppm for 